\title{
Review: healthcare decision aids improve knowledge, decrease decisional conflict, and increase active participation
}

O'Connor AM, Rostom A, Fiset Vet al. Decision aids for patients facing health treatment or screening decisions: systematic review. BMJ 1999 Sep 18;319:731-4.

QUESTION: Do decision aids (interventions providing information on options and outcomes designed to help people make specific and deliberate choices related to their health) used as adjuncts to counselling by healthcare practitioners, improve decision making and outcomes for people making treatment or screening decisions?

\section{Data sources}

Studies were identified by searching Medline (1966 to April 1998), EMBASE/Excerpta Medica (1980 to November 1998), PsycINFO (1979 to March 1998), CINAHL (1983 to February 1998), Aidsline (1980-98), CancerLit (1983 to April 1998), Cochrane Library (1998, Issue 4), personal files, and 3 healthcare journals.

\section{Study selection}

Randomised controlled trials were selected if decision aids were compared with controls or alternative interventions and participants were $>14$ years of age and were making healthcare choices about treatment and screening. Studies were excluded that used hypothetical situations or choices about lifestyle, participation in a clinical trial, advance directives, general education, or compliance issues.

\section{Data extraction}

Data were extracted on numbers of participants, choices considered, and outcomes (knowledge, decisional conflict, decisions made, satisfaction, anxiety, and participation in decision making).

\section{Main results}

17 studies (3 screening and 8 treatment decisions) met the inclusion criteria. Decision aids improved knowledge scores (weighted mean difference in point score, 19 out of $100,95 \%$ CI 14 to 25 when decision aids were compared with usual care and 3 out of 100 , CI 0.7 to 5 when more intensive aids were compared with less intensive aids) (4 studies for both comparisons). Decisional conflict was decreased slightly in 1 of 2 studies comparing decision aids with usual care and 2 of 2 studies comparing intensities of decision aids. Decision aids reduced preferences for more major invasive surgery (relative risk [RR] 0.74, CI 0.6 to $0.9,4$ studies) and for prostate specific antigen testing (2 of 3 studies but no significant effect when results were pooled). 1 study showed increased preferences for hepatitis B vaccine (RR 1.76, CI 1.3 to 2.5) but no other changes in preferences were found for circumcision of newborn infants, screening for breast cancer genes, prenatal testing, dental surgery, or hormone replacement therapy. No pooled difference was shown for satisfaction with the decision or the process of decision making (3 studies) or patient anxiety (4 studies). 3 studies showed an increase in the proportion of participants who assumed a more active role in decision making (RR 2.27, CI 1.3 to 4.0).

\section{Conclusion}

Decision aids improve knowledge, decrease decisional conflict, and increase participation in the decision making process for people who must make healthcare treatment and screening decisions, but the effects on outcomes of decisions remain unclear.

\section{COMMENTARY}

Patient participation in treatment decision making is increasingly encouraged ${ }^{1}$; hence, aids to such decision making are becoming more common. The systematic review by O'Connor et al attempts to determine the effect of such decision aids on several patient related outcomes.

The authors reviewed 17 studies, which covered a wide range of situations and clinical conditions. This diversity is a limitation because, as the authors point out, patients' responses in different situations may not be comparable Further complicating this review is the inconsistency in types of decision support techniques and their definitions across studies.

The review's most noteworthy conclusion is that an individual's involvement in treatment decision making seems, on balance, to be beneficial. Despite variability in types of decision aids and clinical conditions, the trials were consistent in showing that decision aids improve participants' knowledge about options, reduce their decisional conflict, and stimulate them to take a more active role in decision making without increasing their anxiety. The largest and most consistent benefit of decision aids was improved knowledge of options and outcomes, which emphasises the need for healthcare practitioners to ensure that patients adequately comprehend the options and outcomes to make informed decisions.

Much of the research completed to date has focused on the process of treatment decision making and on the factors that facilitate or hamper patients' participation in this process. ${ }^{2}$ Many important questions remain unanswered and, as the authors themselves acknowledge, more information about these fundamental aspects is required before the effects of decision aids can be appropriately examined. Ann-Louise Caress, RGN, RHV, PhD Lecturer, School of Nursing Midwifery and Health Visiting University of Manchester Manchester, $U K$

1 Richards T. Patients' priorities [editorial]. BMJ 1999;318:277.

2 Coulter A. Partnerships with patients: the pros and cons of shared clinical decision-making. J Health Serv Res Policy 1997;2:112-21. 